\title{
National Study on the Contribution of Family Physicians to the US Emergency Physician Workforce in 2020
}

\author{
Christopher L. Bennett, MD, MA, W. Anthony Gerard, MD, John S. Cullen, MD, \\ Janice A. Espinola, MPH, Ashley F. Sullivan, MS, MPH, Carson E. Clay, BA, and \\ Carlos A. Camargo, Jr., MD, DrPH
}

Background: Family physicians provide a sizable portion of emergency care in the United States. However, there is limited work characterizing this population.

Methods: We completed a cross-sectional analysis of the 2020 American Medical Association Physician Masterfile that was inclusive of all clinically active physicians who designated emergency medicine as their primary or secondary specialty and had family medicine residency training and/or family medicine board certification. We used Accreditation Council for Graduate Medical Education information to determine family medicine residency training and data from the American Board of Medical Specialties to determine family medicine board certification status. We calculated physician density using US Census Bureau estimates; urban-rural assignments were based on Urban Influence Codes.

Results: We identified 4354 clinically active emergency physicians ( $9 \%$ of the overall emergency physician workforce). Of these, a majority were male $(\mathbf{8 8} \%)$ and completed their training at least 20 years ago (84\%), and a majority (59\%) reported emergency medicine as their primary specialty. There is notable variation in physician density per 100,000 US population, and these densities declined compared with prior estimates from 2008.

Conclusions: We find that family physicians represent a sizable portion of the overall emergency physician workforce despite decreases in physician densities across the United States. ( $\mathrm{J}$ Am Board Fam Med 2021;34:1221-1228.)

Keywords: Cross-Sectional Studies, Delivery of Health Care, Emergency Medicine, Family Medicine, Family Physicians, Workforce

\section{Introduction}

The United States currently has a shortage of emergency physicians (EPs), ${ }^{1}$ particularly in rural

This article was externally peer reviewed.

Submitted 16 April 2021; revised 5 July 2021; accepted 8 July 2021.

From Department of Emergency Medicine, Stanford University Medical Center, Palo Alto, CA (CLB); Staff Emergency Physician, Lebanon, PA (WAG); Providence Valdez Medical Center, Valdez, AK (JSC); Department of Emergency Medicine, Massachusetts General Hospital, Harvard Medical School, Boston, MA (JAE, AFS, CEC, CAC). Conflict of interest: None.

Author Contributions: Bennett, Espinola, and Camargo full access to the data; Bennett and Camargo take responsibility for the integrity of the data and accuracy of the data analysis. Concept and design: Bennett and Camargo. Acquisition of data: Bennett, Espinola, and Camargo. Interpretation of data: all authors. Drafting of the manuscript: Bennett, Espinola, and Camargo. Critical revision of areas. $^{2,3}$ We recently completed an analysis of all clinically active EPs in the United States. We found more EPs compared with 2008 but a decrease in the density of EPs in rural areas. ${ }^{2,3}$ Further, we found that nearly a third of rural EPs reported prior family medicine (FM) residency

the manuscript for important intellectual content: all authors. Statistical analysis and mapping: Espinola and Clay. Administrative, technical, or material support: Camargo. Supervision: Camargo.

Corresponding author: Christopher Bennett, MD, MA, Department of Emergency Medicine, Stanford University Medical Center, 900 Welch Road, Suite 350, Palo Alto, CA 94303 (E-mail: christopher.lee.bennett@gmail.com).

\section{See Related Commentary on} Page 1265. 
training; ${ }^{3}$ FM-trained EPs-who we will refer to as FM EPs-play an important role in emergency care in the rural United States, ${ }^{4,5}$ but this population has not been well characterized. Our goal was to characterize the 2020 population of clinically active FM EPs and identify their geographic distribution.

\section{Methods}

We performed a cross-sectional, secondary analysis of the 2020 American Medical Association (AMA) Physician Masterfile as reported previously; ${ }^{2,3}$ the AMA Masterfile is a comprehensive database that includes every physician with a medical license. ${ }^{2,3,6}$ The FM EP population of interest was all clinically active physicians who designated emergency medicine $(\mathrm{EM})$ as their primary or secondary specialty and had FM residency training and/or FM board certification. Residents and those not clinically active (eg, primarily researchers, teaching faculty, nonclinical administrators, or physicians who retired or semiretired) were excluded given our interest in clinically active EPs. ${ }^{2,3}$ We used Accreditation Council for Graduate Medical Education (ACGME) information to determine FM residency training and American Board of Medical Specialties (ABMS) data to determine FM board certification status.

As described previously, physician location was classified into US Census divisions. ${ }^{2,3}$ Corresponding population sizes were classified using US Office of Management and Budget groups. ${ }^{2,3}$ Urban Influence Codes were used to assign each EP's county location (urban, large rural, or small rural); FM EP population density was calculated using county-level 2019 Census Bureau population estimates. ${ }^{2,3}$

Data were analyzed using Stata 15.1 (StataCorp; College Station, TX). First, we grouped FM EPs by board certification status: FM board certification with or without EM board certification, EM board certification, board certification but not in FM or $\mathrm{EM}$, and no board certification. In the primary analysis, those with both FM and EM board certification ( $n=736$ ) were classified under the FM board certification group. In a sensitivity analysis, we split the FM board certification group by those with isolated FM board certification versus those with dual FM and EM board certification. Second, we examined FM EPs' characteristics by sex. Physician population density was mapped using ArcMap 10.6.1 (ESRI; Redlands, CA). The MassGeneral Brigham (Boston) Institutional
Review Board determined that this study met criteria for exemption. This study followed the Strengthening in the Reporting of Observational Studies in Epidemiology guidelines for observational studies. ${ }^{7}$

\section{Results}

We identified 4354 EPs with FM training and/or FM board certification ( $9 \%$ of the overall EP workforce). The majority (83\%) were FM board certified, but a small subset lacked either FM or EM board certification (1\%) or had no board certification (7\%) (Table 1). Overall, a majority were male $(88 \%)$ and located in urban areas (79\%). Most (84\%) completed their training at least 20 years ago, and a majority $(59 \%)$ reported $\mathrm{EM}$ as their primary specialty. EPs in small rural areas were similarly aged (median age of 65 years [interquartile range (IQR) 60 to 69 years]) as EPs in large rural areas (65 [58 to 69]) and urban areas (65 [58 to 69]) but more often FM trained (small rural: $92 \%$, large rural: $87 \%$, urban: $82 \%, P<.001$ ), had completed their training at least 20 years ago (small rural: $89 \%$, large rural: $87 \%$, urban: $84 \%, P=.003$ ), and more frequently reported FM board certification (small rural: $87 \%$, large rural: $86 \%$, urban: $82 \%, P<.001$ ). The majority of all groups reported EM as their primary specialty (small rural: $53 \%$, large rural: $58 \%$, urban: $60 \%, P=.04)$.

FM EPs with isolated FM board certification (compared with those with dual FM and EM board certification) were younger (63 [58 to 67] vs 69 [66 to 72]), more often female (13\% vs $9 \%$ ), more often in rural (both large [14\% vs $10 \%]$ and small rural $[10 \%$ vs $3 \%]$ ) areas, and less likely to report EM as their primary specialty (49\% vs $82 \%$ ) (Appendix Table 1). Stratified by sex, female EPs (compared with male EPs) were younger (62 [54 to 67] vs 65 [59 to 69]) but had similar geographic distribution (eg, by US Census division) and were similarly located in urban (77\% vs $79 \%$ ) and rural (large rural [14\% vs $13 \%]$ and small rural $[9 \%$ vs $9 \%]$ ) areas. Additional comparisons of FM EPs stratified by sex are presented in Table 2 .

We also identified notable variation in FM EP density by area per 100,000 US population (total [1.3], urban [1.2], large rural [2.1], and small rural [2.0]). Given prior 2008 data, this represents FM EP density decreases in all areas (total $[-0.5]$, urban $[-0.4]$, large rural $[-0.5]$, and small rural $[-0.4]$ areas). ${ }^{3}$ Physician density is presented in Figure 1 (EPs per 100,000 population by county) and Figure 2 (percent FM EPs among all EPs by county) for all clinically active FM EPs. Although the panels 
Table 1. Comparison of Family Medicine Trained and/or Board Certified Emergency Physicians by Board

\section{Certification}

\begin{tabular}{|c|c|c|c|c|c|c|c|c|c|c|c|}
\hline \multirow{2}{*}{ Characteristics } & \multicolumn{2}{|c|}{$\begin{array}{c}\text { All Family } \\
\text { Medicine EPs, } \\
\mathrm{n}=4354\end{array}$} & \multicolumn{2}{|c|}{$\begin{array}{c}\text { Family } \\
\text { Medicine Board } \\
\text { Certification, } \\
\mathrm{n}=3596 \\
\end{array}$} & \multicolumn{2}{|c|}{$\begin{array}{c}\text { General } \\
\text { Emergency } \\
\text { Medicine Board } \\
\text { Certification, } \\
\mathrm{n}=388 \\
\end{array}$} & \multicolumn{2}{|c|}{$\begin{array}{l}\text { Neither Family } \\
\text { Medicine nor } \\
\text { Emergency } \\
\text { Medicine Board } \\
\text { Certification, } \\
\mathrm{n}=51 \\
\end{array}$} & \multicolumn{2}{|c|}{$\begin{array}{c}\text { No Board } \\
\text { Certification, } \\
n=319\end{array}$} & \multirow[b]{2}{*}{$P$ Value* } \\
\hline & $\mathrm{n}$ & $\%$ & $\mathrm{n}$ & $\%$ & $\mathrm{n}$ & $\%$ & $\mathrm{n}$ & $\%$ & $\mathrm{n}$ & $\%$ & \\
\hline \multicolumn{12}{|l|}{ Demographics } \\
\hline Age, y (median [IQR]) & 4354 & $65(59$ to 69$)$ & 3596 & $64(59$ to 69$)$ & 388 & $66(58$ to 70$)$ & 51 & $67(62$ to 71$)$ & 319 & 65 (60 to 71$)$ & $<0.001$ \\
\hline Age categories, y & & & & & & & & & & & $<0.001$ \\
\hline 25 to 44 & 138 & 3 & 110 & 3 & 14 & 4 & 1 & 2 & 13 & 4 & \\
\hline 45 to 64 & 1991 & 46 & 1701 & 47 & 135 & 35 & 21 & 41 & 134 & 42 & \\
\hline$\geq 65$ & 2225 & 51 & 1785 & 50 & 239 & 62 & 29 & 57 & 172 & 54 & \\
\hline Female sex & 531 & 12 & 429 & 12 & 56 & 14 & 5 & 10 & 41 & 13 & 0.48 \\
\hline $\begin{array}{l}\text { International medical } \\
\text { graduate }\end{array}$ & 701 & 16 & 589 & 16 & 33 & 9 & 8 & 16 & 71 & 22 & $<0.001$ \\
\hline \multicolumn{12}{|l|}{ Geography } \\
\hline US Census division & & & & & & & & & & & $<0.001$ \\
\hline New England & 175 & 4 & 149 & 4 & 15 & 4 & 2 & 4 & 9 & 3 & \\
\hline Mid Atlantic & 341 & 8 & 281 & 8 & 34 & 9 & 4 & 8 & 22 & 7 & \\
\hline East North Central & 590 & 14 & 496 & 14 & 36 & 9 & 9 & 18 & 49 & 16 & \\
\hline West North Central & 391 & 9 & 349 & 10 & 19 & 5 & 2 & 4 & 21 & 7 & \\
\hline South Atlantic & 903 & 21 & 728 & 20 & 100 & 26 & 8 & 16 & 67 & 22 & \\
\hline East South Central & 415 & 10 & 341 & 10 & 28 & 7 & 5 & 10 & 41 & 13 & \\
\hline West South Central & 586 & 14 & 491 & 14 & 33 & 9 & 10 & 20 & 52 & 17 & \\
\hline Mountain & 366 & 8 & 305 & 9 & 40 & 10 & 5 & 10 & 16 & 5 & \\
\hline Pacific & 561 & 13 & 441 & 12 & 80 & 21 & 6 & 12 & 34 & 11 & \\
\hline MSA population size & & & & & & & & & & & $<0.001$ \\
\hline$\geq 1,000,000$ & 1722 & 40 & 1367 & 38 & 203 & 52 & 26 & 51 & 126 & 40 & \\
\hline 250,000 to 999,999 & 939 & 22 & 801 & 22 & 74 & 19 & 9 & 18 & 55 & 17 & \\
\hline 100,000 to 249,999 & 474 & 11 & 398 & 11 & 38 & 10 & 4 & 8 & 34 & 11 & \\
\hline$<100,000$ & 65 & 1 & 54 & 2 & 4 & 1 & 2 & 4 & 5 & 2 & \\
\hline Unknown & 1154 & 27 & 976 & 27 & 69 & 18 & 10 & 20 & 99 & 31 & \\
\hline Urban influence & & & & & & & & & & & $<0.001$ \\
\hline Urban & 3402 & 79 & 2781 & 78 & 340 & 88 & 40 & 78 & 241 & 76 & \\
\hline Large rural & 560 & 13 & 479 & 13 & 33 & 9 & 7 & 14 & 41 & 13 & \\
\hline Small rural & 372 & 9 & 322 & 9 & 12 & 3 & 4 & 8 & 34 & 11 & \\
\hline \multicolumn{12}{|l|}{ Training } \\
\hline Residency & & & & & & & & & & & $<0.001$ \\
\hline Emergency medicine & 403 & 9 & 176 & 5 & 200 & 52 & 3 & 6 & 24 & 8 & \\
\hline Family medicine & 3646 & 84 & 3181 & 88 & 162 & 42 & 21 & 41 & 282 & 88 & \\
\hline Internal medicine & 66 & 2 & 29 & 0.8 & 14 & 4 & 19 & 37 & 4 & 1 & \\
\hline Pediatrics & 11 & 0.3 & 6 & 0.2 & 3 & 0.8 & 2 & 4 & 0 & 0 & \\
\hline Surgery & 62 & 1 & 38 & 1 & 9 & 2 & 6 & 12 & 9 & 3 & \\
\hline Internship only & 58 & 1 & 58 & 2 & 0 & 0 & 0 & 0 & 0 & 0 & \\
\hline Other residency & 13 & 0.3 & 13 & 0.4 & 0 & 0 & 0 & 0 & 0 & 0 & \\
\hline None & 95 & 2 & 95 & 3 & 0 & 0 & 0 & 0 & 0 & 0 & \\
\hline
\end{tabular}




\begin{tabular}{|c|c|c|c|c|c|c|c|c|c|c|c|}
\hline \multirow{2}{*}{ Characteristics } & \multicolumn{2}{|c|}{$\begin{array}{c}\text { All Family } \\
\text { Medicine EPs, } \\
\mathrm{n}=4354\end{array}$} & \multicolumn{2}{|c|}{$\begin{array}{c}\text { Family } \\
\text { Medicine Board } \\
\text { Certification, } \\
\mathrm{n}=3596\end{array}$} & \multicolumn{2}{|c|}{$\begin{array}{c}\text { General } \\
\text { Emergency } \\
\text { Medicine Board } \\
\text { Certification, } \\
\mathrm{n}=388\end{array}$} & \multicolumn{2}{|c|}{$\begin{array}{c}\text { Neither Family } \\
\text { Medicine nor } \\
\text { Emergency } \\
\text { Medicine Board } \\
\text { Certification, } \\
\mathrm{n}=51\end{array}$} & \multicolumn{2}{|c|}{$\begin{array}{c}\text { No Board } \\
\text { Certification, } \\
\mathrm{n}=319\end{array}$} & \multirow[b]{2}{*}{$P$ Value } \\
\hline & $\mathrm{n}$ & $\%$ & $\mathrm{n}$ & $\%$ & $\mathrm{n}$ & $\%$ & $\mathrm{n}$ & $\%$ & $\mathrm{n}$ & $\%$ & \\
\hline $\begin{array}{l}\text { Years since completed } \\
\text { medical training }\end{array}$ & & & & & & & & & & & 0.006 \\
\hline$<5$ & 44 & 1 & 34 & 1 & 1 & 0.3 & 1 & 2 & 8 & 3 & \\
\hline 5 to 9 & 98 & 2 & 79 & 2 & 13 & 3 & 0 & 0 & 6 & 2 & \\
\hline 10 to 19 & 520 & 12 & 415 & 12 & 66 & 17 & 4 & 8 & 35 & 11 & \\
\hline$\geq 20$ & 3597 & 84 & 2973 & 85 & 308 & 79 & 46 & 90 & 270 & 85 & \\
\hline $\begin{array}{l}\text { Primary specialty of } \\
\text { emergency medicine }\end{array}$ & 2563 & 59 & 2004 & 56 & 363 & 94 & 9 & 18 & 187 & 59 & $<0.001$ \\
\hline
\end{tabular}

EPs, emergency physicians; IQR, interquartile range; MSA, metropolitan statistical area.

*Bivariate comparisons were tested using Kruskal-Wallis, $\chi^{2}$, and Fisher's exact tests, as appropriate.

demonstrate FM EPs across the country, they seem most prevalent at the edges of our previously reported band of underserved states (from North Dakota to Texas)— the "EP desert."

\section{Discussion}

We find that FM EPs represent a sizable portion of the clinically active EP workforce and most are male and in urban areas. Although less than a quarter were located in rural areas, the rural proportions presented here are higher than those of the overall EP population (we recently reported that only a minority of all EPs are in either large [6\%] or small [3\%] rural areas). ${ }^{3}$ We also found that FM EPs are older than the overall EP workforce and FM EPs in small and large rural areas are older than counterparts from the overall EP workforce. ${ }^{3}$ Further, compared with $2008,{ }^{3}$ we find decreases in FM EP density across the United States (overall, urban, large rural, and small rural). Our findings characterize a rural, predominately male subpopulation of older FM EPs, who more often have isolated FM board certification, and who provide care in already underserved areas of the country where overall EP density is declining_-EP deserts."

However, our work has limitations. ${ }^{2,3}$ First, our study was limited to physicians who self-designated EM as their primary or secondary specialty. Family physicians who provide emergency care in the United States may not necessarily identify as EPs. As such, we likely slightly underestimate the number of actual FM EPs. ${ }^{4,5}$ Second, we are unable to account for the contributions of osteopathic EPs. Data are linked only to ACGME and ABMS; there is no equivalent osteopathic database. ${ }^{2,3}$ This is a previously noted limitation. ${ }^{2,3}$ Despite this, our work is still the most current and comprehensive study on clinically active FM EPs in the United States. Third, we are unable to account for the impact graduating EM residents will have on the FM EP workforce. We recently demonstrated increasing densities of EPs in urban areas; ${ }^{3} \mathrm{EM}$ resident graduates have historically not taken jobs in rural areas. ${ }^{8}$ This absence of EM residency-trained EPs has likely contributed to the large share of FM EPs providing emergency care in more remote areas.

COVID-19 has significantly impacted the EP workforce. ${ }^{9}$ Fewer jobs, ${ }^{9}$ an increase in the number of EM residency programs, ${ }^{10}$ and a potential surplus of EPs by $2030^{11}$ may collectively mean that new graduates joining the workforce will now relocate to areas and work in rural settings where FM EPs predominate.

Efforts that promote an increased physician presence in rural America are required. As FM EPs leave the workforce, "EP deserts" will persist and likely expand. However, addressing the need for rural EPs will require concerted effort. ${ }^{2,3}$ Incentivizing relocation efforts through loan repayment and expanding the number of rural EM rotations have been suggested. ${ }^{12,13}$ In the absence of any significant change, we anticipate that declining EP densities (both all EPs and FM EPs) in rural America will contribute to 
Table 2. Comparison of Family Medicine Emergency Physicians by Sex, $\mathrm{n}=4354$

\begin{tabular}{|c|c|c|c|c|c|}
\hline \multirow[b]{3}{*}{ Characteristics } & \multicolumn{4}{|c|}{ All Family Medicine EPs, } & \multirow[b]{3}{*}{$P$ Value* } \\
\hline & \multicolumn{2}{|c|}{ Male $(\mathrm{n}=3823)$} & \multicolumn{2}{|c|}{ Female $(\mathrm{n}=531)$} & \\
\hline & $\mathrm{n}$ & $\%$ & $\mathrm{n}$ & $\%$ & \\
\hline \multicolumn{6}{|l|}{ Demographics } \\
\hline Age, y (median [IQR]) & 3823 & $65(59$ to 69$)$ & 531 & $62(54$ to 67$)$ & $<0.001$ \\
\hline Age categories, y & & & & & $<0.001$ \\
\hline 25 to 44 & 101 & 3 & 37 & 7 & \\
\hline 45 to 64 & 1691 & 44 & 300 & 57 & \\
\hline$\geq 65$ & 2031 & 53 & 194 & 37 & \\
\hline International medical graduate & 630 & 16 & 71 & 13 & 0.07 \\
\hline \multicolumn{6}{|l|}{ Geography } \\
\hline US Census division & & & & & 0.87 \\
\hline New England & 148 & 4 & 27 & 5 & \\
\hline Mid Atlantic & 302 & 8 & 39 & 7 & \\
\hline East North Central & 519 & 14 & 71 & 13 & \\
\hline West North Central & 340 & 9 & 51 & 10 & \\
\hline South Atlantic & 789 & 21 & 114 & 22 & \\
\hline East South Central & 371 & 10 & 44 & 8 & \\
\hline West South Central & 512 & 13 & 74 & 14 & \\
\hline Mountain & 321 & 8 & 45 & 9 & \\
\hline Pacific & 498 & 13 & 63 & 12 & \\
\hline MSA population size & & & & & 0.03 \\
\hline$\geq 1,000,000$ & 1503 & 39 & 219 & 41 & \\
\hline 250,000 to 999,999 & 815 & 21 & 124 & 23 & \\
\hline 100,000 to 249,999 & 433 & 11 & 41 & 8 & \\
\hline$<100,000$ & 52 & 1 & 13 & 2 & \\
\hline Unknown & 1020 & 27 & 134 & 25 & \\
\hline Urban influence & & & & & 0.77 \\
\hline Urban & 2993 & 79 & 409 & 77 & \\
\hline Large rural & 487 & 13 & 73 & 14 & \\
\hline Small rural & 325 & 9 & 47 & 9 & \\
\hline \multicolumn{6}{|l|}{ Training } \\
\hline Residency & & & & & $<0.001$ \\
\hline Emergency medicine & 322 & 8 & 81 & 15 & \\
\hline Family medicine & 3232 & 85 & 414 & 78 & \\
\hline Internal medicine & 59 & 2 & 7 & 1 & \\
\hline Pediatrics & 8 & 0.2 & 3 & 0.6 & \\
\hline Surgery & 57 & 1 & 5 & 0.9 & \\
\hline Internship only & 57 & 1 & 1 & 0.2 & \\
\hline Other residency & 12 & 0.3 & 1 & 0.2 & \\
\hline None & 76 & 2 & 19 & 4 & \\
\hline Years since completed medical training & & & & & $<0.001$ \\
\hline$<5$ & 29 & 0.8 & 15 & 3 & \\
\hline 5 to 9 & 76 & 2 & 22 & 4 & \\
\hline 10 to 19 & 421 & 11 & 99 & 19 & \\
\hline$\geq 20$ & 3221 & 86 & 376 & 73 & \\
\hline Primary specialty of emergency medicine & 2244 & 59 & 319 & 60 & 0.55 \\
\hline
\end{tabular}

EPs, emergency physicians; IQR, interquartile range; MSA, metropolitan statistical area.

*Bivariate comparisons were tested using Kruskal-Wallis, $\chi^{2}$, and Fisher's exact tests, as appropriate. 
Figure 1. Family medicine emergency physician density per 100,000 population by county.

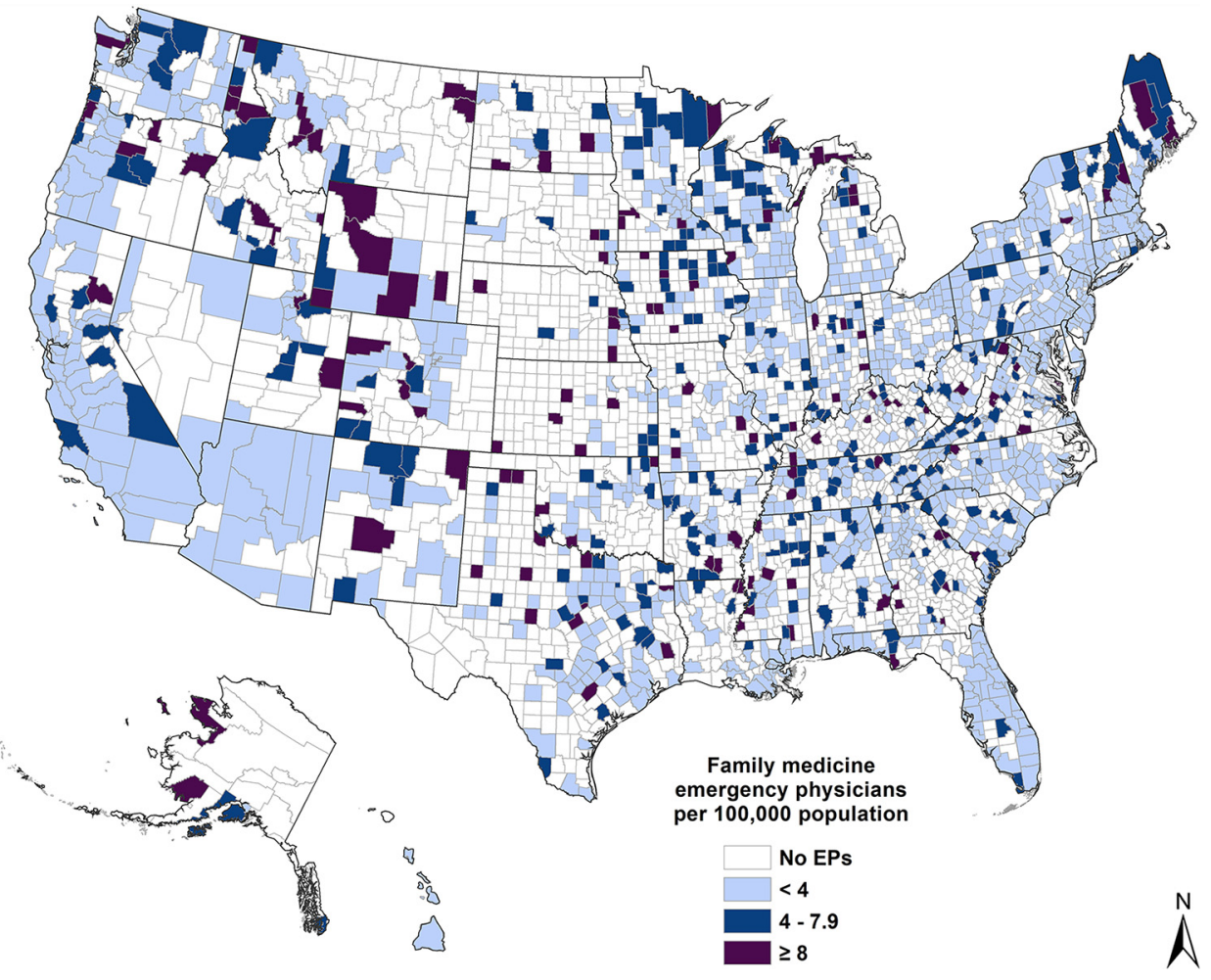

Figure 2. Family medicine emergency physician percent among all clinically active emergency physicians by county.

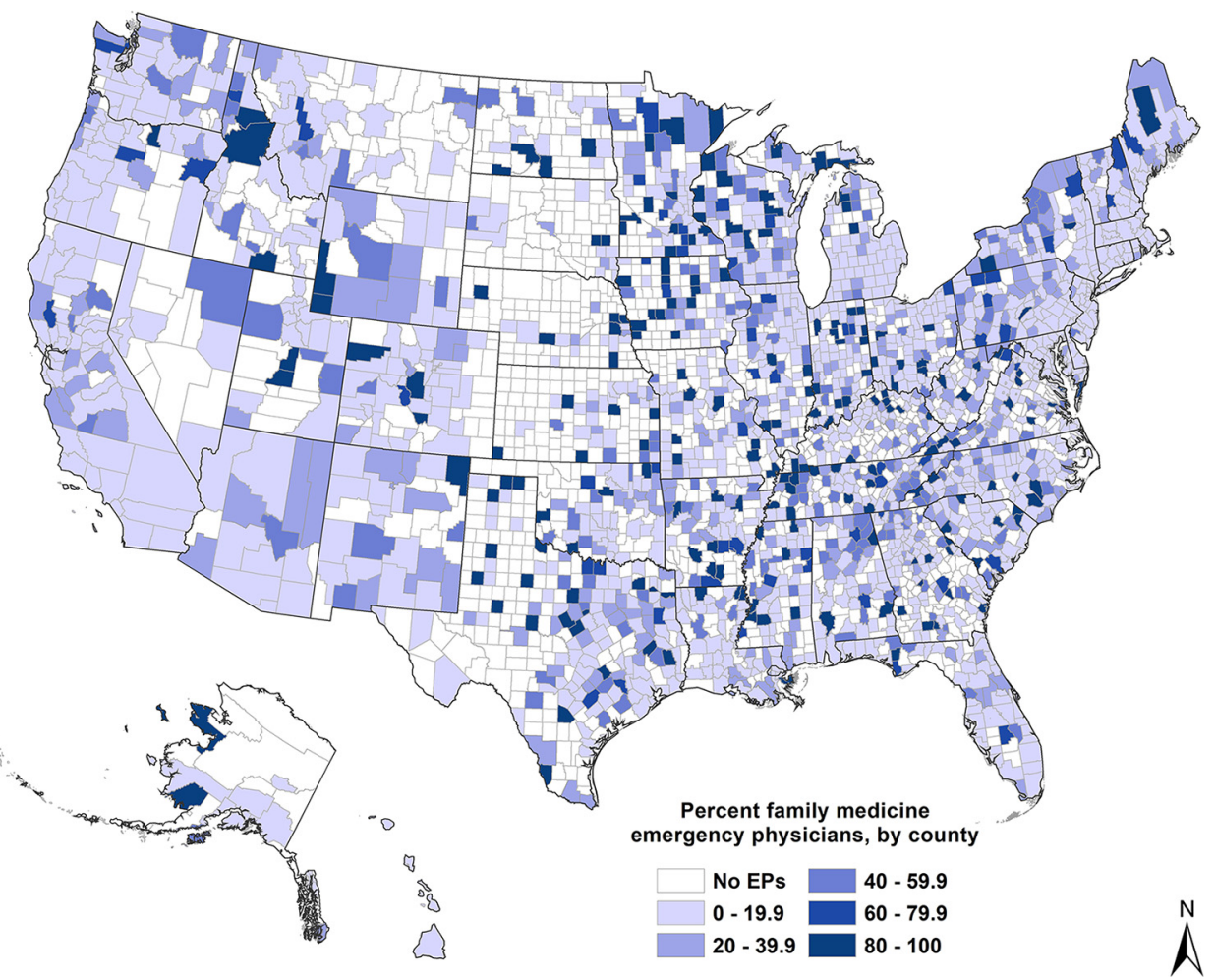


an accelerated used of nonphysician models of care with an increased presence of nurse practitioners, physician assistants, and/or telehealth. ${ }^{14,15}$

To see this article online, please go to: http://jabfm.org/content/ 34/6/1221.full.

\section{References}

1. Reiter M, Wen LS, Allen BW. The emergency medicine workforce: profile and projections. J Emerg Med 2016;50:690-3.

2. Ginde AA, Sullivan AF, Camargo CA, Jr. National study of the emergency physician workforce, 2008. Ann Emerg Med 2009;54:349-59.

3. Bennett CL, Sullivan AF, Ginde AA, et al. National study of the emergency physician workforce, 2020. Ann Emerg Med 2020;76:695-708.

4. Peterson LE, Puffer JC, Nasim U, Petterson S, Newton WP. Family physicians' contributions to rural emergency care and urban urgent care. J Am Board Fam Med 2019;32:295-6.

5. Banks GC, Gerard AW, Yu K, Bullock KA, Members, AAFP Member Interest Group Emergency Medicine/Urgent Care. Family physicians play integral role in emergency medicine. Ann Fam Med 2017;15:82-86.

6. AMA Physician Masterfile. American Medical Association. Available from: https://www.amaassn.org/about/masterfile/ama-physician-masterfile. Accessed March 21, 2021.

7. Von Elm E, Altman DG, Egger M, Pocock SJ, Gøtzsche PC, Vandenbroucke JP, STROBE Initiative. The Strengthening the Reporting of Observational Studies in Epidemiology (STROBE) statement: guidelines for reporting observational studies. Lancet 2007;370:1453-7.

8. Wadman MC, Clark TR, Kupas DF, et al. Rural clinical experiences for emergency medicine residents: a curriculum template. Acad Emerg Med 2012;19: 1287-93.

9. Washington Post; January 4, 2021. Young ER doctors risk their lives on the pandemic's front lines. But they struggle to find jobs. Available from: https:// www.washingtonpost.com/health/2021/01/04/erdoctors-covid-jobs/. Accessed March 30, 2021.

10. ACGME Data Resource Book. Available from: https://www.acgme.org/About-Us/Publicationsand-Resources/Graduate-Medical-Education-DataResource-Book. Accessed March 30, 2021.

11. Reiter M, Allen BW. The emergency medicine workforce: shortage resolving, future surplus expected. J Emerg Med 2020;58:198-202.

12. Renner DM, Westfall JM, Wilroy LA, Ginde AA. The influence of loan repayment on rural healthcare provider recruitment and retention in Colorado. Rural Remote Health 2010;10:1605.

13. Talley BE, Ann Moore S, Camargo CA, Rogers J, Ginde AA. Availability and potential effect of rural rotations in emergency medicine residency programs. Acad Emerg Med 2011;18:297-300.

14. Brown DF, Sullivan AF, Espinola JA, et al. Continued rise in the use of midlevel providers in US emergency departments, 1993 to 2009. Int J Emerg Med. 2012;21:1-5.

15. Zachrison KS, Boggs KM, Hayden E, et al. Understanding barriers to telemedicine implementation in rural emergency departments. Ann Emerg Med 2020;75:392-9. 
Appendix Table 1. Comparison of Family Medicine Emergency Physicians with Isolated Family Medicine or Dual Family Medicine and Emergency Medicine Board Certification, $n=3596$

\begin{tabular}{|c|c|c|c|c|c|}
\hline \multirow[b]{2}{*}{ Characteristics } & \multicolumn{2}{|c|}{$\begin{array}{l}\text { Isolated FM Board Certification } \\
\qquad(\mathrm{n}=2860)\end{array}$} & \multicolumn{2}{|c|}{$\begin{array}{l}\text { Dual FM and EM Board } \\
\text { Certification }(\mathrm{n}=736)\end{array}$} & \multirow[b]{2}{*}{$P$ Value* } \\
\hline & $\mathrm{n}$ & $\%$ & $\mathrm{n}$ & $\%$ & \\
\hline \multicolumn{6}{|l|}{ Demographics } \\
\hline Age, y (median [IQR]) & 2860 & $63(58$ to 67$)$ & 736 & 69 (66 to 72$)$ & $<0.001$ \\
\hline Age categories, y & & & & & $<0.001$ \\
\hline 25 to 44 & 93 & 3 & 17 & 2 & \\
\hline 45 to 64 & 1573 & 55 & 128 & 17 & \\
\hline$\geq 65$ & 1194 & 42 & 591 & 80 & \\
\hline Female sex & 364 & 13 & 65 & 9 & 0.004 \\
\hline International medical graduate & 512 & 18 & 77 & 10 & $<0.001$ \\
\hline \multicolumn{6}{|l|}{ Geography } \\
\hline US Census division & & & & & $<0.001$ \\
\hline New England & 108 & 4 & 41 & 6 & \\
\hline Mid Atlantic & 216 & 8 & 65 & 9 & \\
\hline East North Central & 395 & 14 & 101 & 14 & \\
\hline West North Central & 303 & 11 & 46 & 6 & \\
\hline South Atlantic & 583 & 20 & 145 & 20 & \\
\hline East South Central & 295 & 10 & 46 & 6 & \\
\hline West South Central & 426 & 15 & 65 & 9 & \\
\hline Mountain & 216 & 8 & 89 & 12 & \\
\hline Pacific & 306 & 11 & 135 & 18 & \\
\hline MSA population size & & & & & $<0.001$ \\
\hline$\geq 1,000,000$ & 1036 & 36 & 331 & 45 & \\
\hline 250,000 to 999,999 & 617 & 22 & 184 & 25 & \\
\hline 100,000 to 249,999 & 310 & 11 & 88 & 12 & \\
\hline$<100,000$ & 42 & 1 & 12 & 2 & \\
\hline Unknown & 855 & 30 & 121 & 16 & \\
\hline Urban influence & & & & & $<0.001$ \\
\hline Urban & 2142 & 75 & 639 & 87 & \\
\hline Large rural & 409 & 14 & 70 & 10 & \\
\hline Small rural & 297 & 10 & 25 & 3 & \\
\hline \multicolumn{6}{|l|}{ Training } \\
\hline Residency & & & & & $<0.001$ \\
\hline Emergency medicine & 29 & 1 & 147 & 20 & \\
\hline Family medicine & 2665 & 93 & 516 & 70 & \\
\hline Internal medicine & 14 & 0.5 & 15 & 2 & \\
\hline Pediatrics & 3 & 0.1 & 3 & 0.4 & \\
\hline Surgery & 26 & 0.9 & 12 & 2 & \\
\hline Internship only & 35 & 1 & 23 & 3 & \\
\hline Other residency & 6 & 0.2 & 7 & 1 & \\
\hline None & 82 & 3 & 13 & 2 & \\
\hline Years since completed medical training & & & & & 0.002 \\
\hline$<5$ & 29 & 1 & 5 & 0.7 & \\
\hline 5 to 9 & 53 & 2 & 26 & 4 & \\
\hline 10 to 19 & 350 & 13 & 65 & 9 & \\
\hline$\geq 20$ & 2346 & 84 & 627 & 87 & \\
\hline Primary specialty of emergency medicine & 1402 & 49 & 602 & 82 & $<0.001$ \\
\hline
\end{tabular}

EM, emergency medicine; FM, family medicine; IQR, interquartile range; MSA, metropolitan statistical area.

*Bivariate comparisons were tested using Kruskal-Wallis, $\chi^{2}$, and Fisher's exact tests, as appropriate. 